\title{
The 2nd Metacarpal Cortical Index as a Simple Screening Tool for Osteopenia
}

\author{
Bhavika Patel, Adeel Aqil, Osman Riaz, Russ Jeffers, David Dickson \\ Bradford Teaching Hospitals NHS Foundation Trust, Duckworth Lane, Bradford, United Kingdom
}

\author{
Corresponding author \\ Bhavika Patel \\ Bradford Teaching Hospitals NHS Foundation \\ Trust, Duckworth Lane, Bradford BD9 6RJ, \\ United Kingdom \\ Tel: +44-750-104-0772 \\ E-mail: patelb493@gmail.com
}

The abstract of this study has been presented at the autumn meeting of the British Society for Surgery of the Hand (BSSH) conference (10 Oct. 2018), and 14th triennial congress of the International Federation of Societies for Surgery of the Hand (IFSSH), 11th triennial congress of the International Federation of Societies for Hand Therapy (IFSHT) (19 Jun. 2019).

Received: September 18, 2020

Revised: October 28, 2020

Accepted: November 1, 2020
Background: Osteopenia is a known risk factor for sustaining skeletal fractures. Prevention of fragility fractures has obvious clinical and economic advantages, however screening all patients using a dual energy X-ray absorptiometry (DXA) is controversial not only because of the cost implications but also because it would potentially involve exposing a percentage of normal patients to unnecessary radiation. We wished to assess whether a simple hand X-ray measuring the 2 nd metacarpal cortical index ( $2 \mathrm{MCl}$ ) could be used as a simple screening tool for identifying patients with osteopenia. Methods: We retrospectively collected the radiographic data of 206 patients who had a simple radiograph of the hand and a DXA scan within one year of each other from our picture archiving and communication system database. The $2 \mathrm{MCl}$ was calculated for all patients. As data was parametric, a Pearson's correlation was performed to assess association between Tscores and $2 \mathrm{MCl}$. Further analysis involved the construction of receiver operating characteristic (ROC) curves to identify a $2 \mathrm{MCl}$ index, which would give the most appropriate sensitivity and specificity values for identifying the presence of osteopenia. Results: A statistically significant and moderate correlation between DXA T-scores and $2 \mathrm{MCl}$ values was found ( $r=0.54, n=206, P<0.001)$. Further ROC curve analysis of normal and osteopenic subjects identified that a $2 \mathrm{MCl}$ of 41.5 had a sensitivity of $100 \%$ and specificity of $53 \%$ for detecting osteopenia. Conclusions: Our results support the use of the $2 \mathrm{MCl}$ as a simple screening tool for identifying patients with osteopenia.

Key Words: Bone diseases, metabolic $\cdot$ Metacarpus $\cdot$ Osteoporosis $\cdot$ Radiography

\section{INTRODUCTION}

Osteoporosis is a metabolic condition in which there is a loss in bone mass, causing bone to become brittle and fracture easily. The current prevalence of osteoporosis is approximately 3 million in the United Kingdom,[1] causing over 300,000 fragility fractures per year.[2] The most common fragility fractures occur at the wrist, vertebra and hip.[3] Every year, hip fractures cause 69,000 admissions in England,[4] costing the National Health Service (NHS) roughly $£ 1.8$ billion in the year 2000. This cost is predicted to rise to $£ 2.2$ billion by 2025.[5] Females aged between 65 and 74 years of age who have sustained a fracture to their wrist are 3 times more likely to develop a hip fracture than in the general population. This figure is 6 times more likely in men.[6] Osteopenia is a precursor to osteoporosis, and thus preventing the progression of osteopenia to osteoporosis may help in preventing fragility fractures. 
Currently, the dual energy X-ray absorptiometry (DXA) scan is the gold standard investigation for osteoporosis in the UK. This is advised in people older than 50 years with a fragility fracture, or younger than 40 years old with a risk factor for fragility fractures.[7] Osteoporosis is said to be a systemic disease affecting the whole skeleton.[8] Bone mineral deficiency leading to osteopenia can result in thinning of cortical bone and the ratio of medullary cancellous to cortical bone changes. The second metacarpal bone is relatively cylindrical and thus $\mathrm{X}$-rays taken from different aspects give relatively consistent cancellous to cortical bone ratios. Therefore, radiographs of the 2 nd metacarpal can be used to calculate the cortical index and in theory may be used to predict deficient bone mineral density (BMD) conditions. Some studies have found that computed tomography (CT) and magnetic resonance imaging scans can be used for screening osteoporosis. $[9,10]$ However, radiographs are readily available, cheap and the 2 nd metacarpal is included in X-rays of the hand, wrist and elbow. So far, there is limited research assessing the value of using plain radiographs to assess for BMD deficiency diseases. However, a recent study has found a significant correlation between BMD and 2nd metacarpal cortical percentage.[11] Another study has also demonstrated a correlation between BMD and mandibular cortical indexes in India.[12] Furthermore, the cylindrical shape of the 2nd metacarpal has been shown to minimise measurement inconsistencies from X-rays taken from slightly different angles.[11]

We therefore aimed to assess if simple radiographs which include the 2nd metacarpal could be used to predict the presence of osteopenia.

\section{METHODS}

\section{Study cohort}

We retrospectively collected radiographic data of 206 patients, who had a simple radiograph of the hand and DXA within one year of each other. These images were obtained from our picture archiving and communication system (PACS) database. Inclusion criteria consisted of patients over the age of 50 years who had both a hand radiograph and DXA between September 2015 and September 2018. Patients with suboptimal X-rays, those with a plaster or metal work obstructing the 2nd metacarpal, and those who had more than 1 year between X-ray and DXA, were excluded. The 222 patients were initially identified, and following application of exclusion criteria, 206 patients remained.

\section{Second metacarpal cortical percentage calculation}

Posteroanterior views of the 2nd metacarpal were used to calculate the 2 nd Metacarpal Cortical Index $(2 \mathrm{MCl})$ by one observer. This was calculated by measuring the diameter of the mid diaphysis at the narrowest point (A). A second measurement was taken from the intramedullary part at the same point (B) (Fig. 1). The formula [(A-B)/A] ${ }^{*} 100$ was used to calculate the $2 \mathrm{MCl}$. The $2 \mathrm{MCl}$ was not re-measured by an independent observer.

\section{Statistical analysis}

The $2 \mathrm{MCl}$ and DXA hip T-score data were initially examined using producing histograms. We found no difference between $2 \mathrm{MCl}$ and spine DXA scores and therefore used hip T-scores only for simplicity. The data visually approximated well to normality curves. To confirm data was parametric, Kolmogorov-Smirnov and Shapiro-Wilk tests were performed and Q-Q plots were constructed.

A Pearson correlation test was performed to measure the

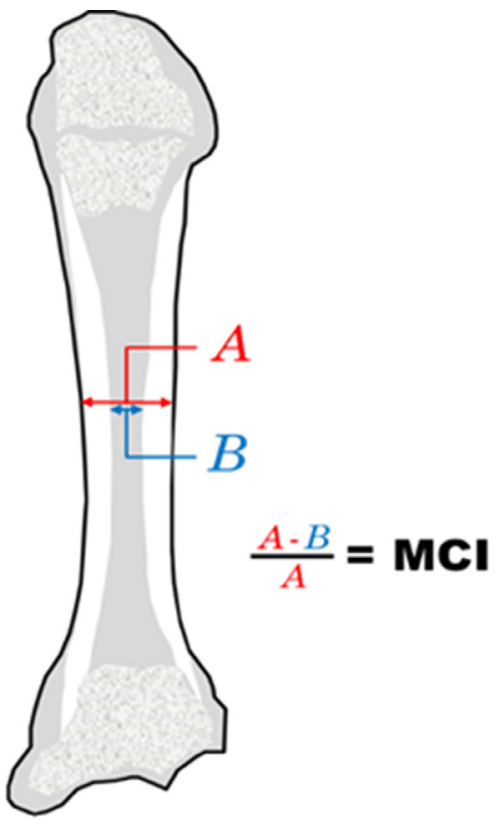

Fig. 1. Demonstration of the calculation of 2nd metacarpal cortical index (2MCl). 


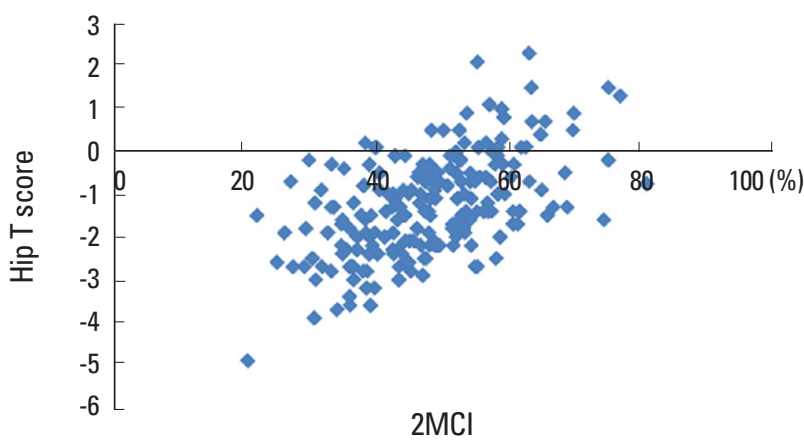

Fig. 2. A scatter graph showing the correlation of 2nd metacarpal cortical index (2MCI) with Hip T score. A significant correlation was found $(r=0.54, n=206, P<0.001)$.

potential association between the $2 \mathrm{MCl}$ and DXA T-Scores. A 2-tailed test was carried out and the $95 \%$ confidence interval was deemed as significant.

Following this receiver operating characteristic (ROC) curves were constructed using DXA T and $2 \mathrm{MCl}$ scores as the test and state variables. As we wished to use the $2 \mathrm{MCl}$ as a potential screening tool for osteopenia, the optimum $2 \mathrm{MCl}$ cut off value was deemed as that where a sensitivity of $100 \%$ was evident with the highest possible specificity.

\section{RESULTS}

This study investigated 206 patients, of which 178 were female and 28 were male. The mean age was $69+10$ years and ranged from 51 to 93 . Eighty-eight patients were osteopenic $(-2.5>$ hip T score $<-1), 33$ were osteoporotic (hip T score $<-2.5)$ and 85 patients had a hip T score greater than -1 . The mean hip T-score was $-1.3+1.2$ (range, -4.9 to 2.3) and mean $2 \mathrm{MCl}$ was $0.48+0.11$ (range, $0.2-0.8$ ). The average time between hand X-ray and DXA scan was 80 days +50 (range, $0-218$ days).

Both $2 \mathrm{MCl}$ and DXA hip T-scores were found to approximate well to a gaussian distribution on drawing histograms, Q-Q plots and on Kolmogorov-Smirnov testing [2MCl: D(206) $=0.03,0.2$ and DXA T score: $\mathrm{D}(206)=0.05,0.2]$.

A Pearson correlation test revealed a moderate and significant correlation between DXA hip T and $2 \mathrm{MCl}$ scores ( $r=0.54, n=206, P<0.001$ ) (Fig. 2). A $2 \mathrm{MCl}$ value of 41.5 showed $100 \%$ sensitivity and $53 \%$ specificity for detecting osteopenia (Fig. 3).

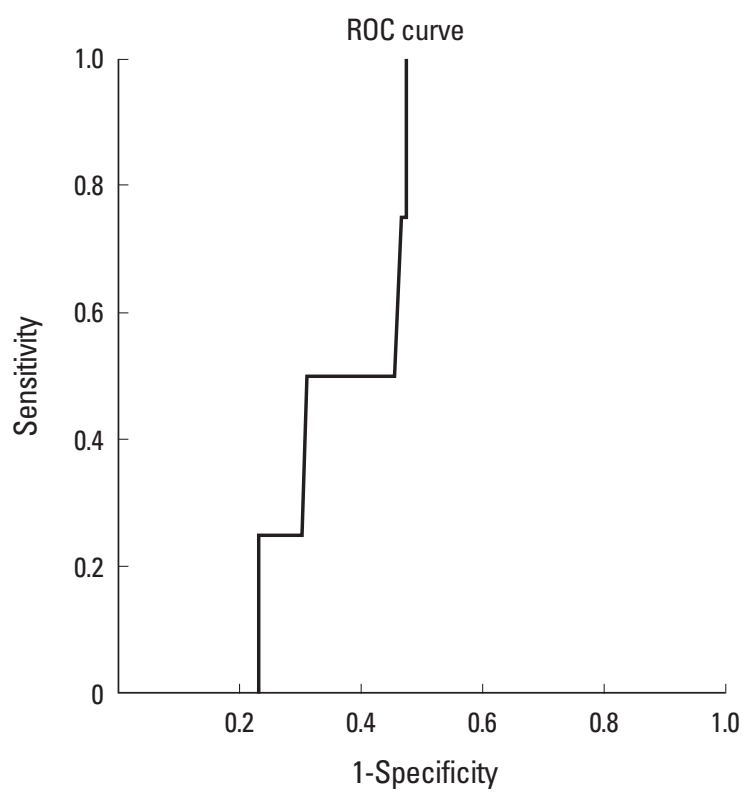

Fig. 3. A receiver operating characteristic $(\mathrm{ROC})$ curve showing sensitivity and specificity values for 2 nd metacarpal cortical index as a predictor for osteopenia (area under curve $=0.63$ ).

\section{DISCUSSION}

This study has demonstrated that the $2 \mathrm{MCl}$ can be used to identify patients with osteopenia. DXA scan is the gold standard for screening for osteopenia and osteoporosis, and a statistically significant correlation was found between DXA hip T-score and $2 \mathrm{MCl}$. A $2 \mathrm{MCl}$ value with $100 \%$ sensitivity and $53 \%$ specificity for osteopenia was found, indicating that this method could be an effective screening tool.

This is the first study to identify a way of using this tool in identifying patients in the pre-osteoporotic state (i.e., osteopenia). This method would be simple, widely available and inexpensive. The results would also be quickly and easily accessible. Measuring the $2 \mathrm{MCl}$ is a recognised technique to identify patients with osteoporosis since 1960. Quantitative evaluations of hand radiographs have been used to investigate the association between cortical thickness and osteoporosis, and a positive relationship between metacarpal bi-cortical thickness and femoral bone density on DXA using PA radiographs of the hand has been demonstrated.[13] However, the precision of this technique was initially poor (coefficient of variation $8 \%-12 \%$ ), due to the use of measuring errors from using non-digital radiographs. The technique was rejuvenated in 2000 with the applica- 
tion of modern computer vision technology.[14] A further study in 2017 found a correlation between the metacarpal cortical percentage with a value of less than $50 \%$ showing $100 \%$ sensitivity and $91 \%$ specificity for osteoporosis.[11] Further studies have demonstrated radiographic quantitative indices as screening tools for osteoporosis,[15-17] and other studies have found a correlation between proximal humeral cortical thickness and DXA scores.[18,19]

Other imaging modalities including $\mathrm{X}$-ray radiogrammetry and quantitative $\mathrm{CT}$ have also been investigated as alternative diagnostic tools to address population screening. [20] Radiogrammetry (DXR) is one way of assessing bone density using computer software to calculate measurements based on simple radiographs.[21] The use of the $2 \mathrm{MCl}$, on the other hand, does not require the use of specialised computer software.

Despite these alternative attempts to evaluate BMD and fracture risk, the method recommended by the World Health Organisation (WHO) for diagnosis of osteopenia and osteoporosis is the measurement of BMD by DXA scans.[22] Currently the DXA method is widely applied and has very reliable outcomes in identifying individuals with low BMD at the hip and vertebrae.

This study has shown a $2 \mathrm{MCl}$ of 41.5 leads to $100 \%$ sensitivity for identifying osteopenic subjects. The ability to identify osteopenia before progression to osteoporosis would allow more expedient treatments and may reduce the incidence of fragility fractures. The accuracy of the radiographic measurements depends on the digital images PACS system with measurement and magnification functions. While we have not tested intra- and inter-observer reliability in this study, digital radiographic measurements have been shown in another study to have a high intraobserver and inter-observer reliability which suggests that this technique would be reproducible.[22]

In many patients, osteopenia and osteoporosis is only diagnosed at the point of the complication of a fragility fracture. Opportunistic radiographic assessment of the $2 \mathrm{MCl}$ when a patient presents to a hand clinic or minor injury unit could identify patients at risk of developing osteoporosis at no extra cost to the NHS. These patients could then be referred to either primary care or fragility clinics to obtain lifestyle advice or to have further screening or treatment as necessary prior to them sustaining a fragility fracture.
In practice, screening tests exhibit false positives and false negatives. Our study shows a $2 \mathrm{MCl}$ of 41.5 leads to $100 \%$ sensitivity with $53 \%$ specificity for osteopenia. This value can allow clinicians to assess the patient and then as appropriate, refer for a DXA scan to detect osteoporosis. The false positive patients during this process can be identified and excluded without receiving treatment.

The limitations of our study included its retrospective nature and study size; a large cohort with multi centred patients would result in a more robust study. While we did not record the ethnicity of our sample, the study was carried out in Bradford Royal Infirmary, which is known to have an ethnically diverse urban population. Future studies could further validate or findings from different patient groups. In order to maximise the number of osteopenia X-rays in our cohort, we limited our selection to patients more than 50 years of age. We accept therefore that our results and conclusions therefore might not be applicable to younger patients. However, it is less likely that younger patients would have BMD related diseases. This study is also limited by not considering patient comorbidities such as rheumatoid arthritis, which may also affect the $2 \mathrm{MCl}$ cortical thickness. We also accept that minor degrees of rotation of the hand during radiographs may marginally affect the ratio described. However, as the metacarpal roughly cylindrical shaped at the mid-level other studies have found rotational malposition of the hand during the X-ray process has found not to have a negligible effect on $2 \mathrm{MCl}$ measurements. There is also the possibility that a patient's BMD/ Tscore may have altered between having X-rays and the DXA scan. However, as part of our inclusion criteria, we limited the interval between the 2 scans in order to reduce this impact. In fact, the average time difference between investigations was less than 3 months and at maximum just over 7 months.

\section{CONCLUSIONS}

This study suggests that the $2 \mathrm{MCl}$ appears to be a simple and effective method for identifying patients with osteopenia, and therefore could be particularly useful in the identification of patients who would benefit from treatment preventing future pathological fractures. External validation on prospective data in the future would be useful to further validate our findings. 


\section{DECLARATIONS}

\section{Ethics approval and consent to participate}

This study was conducted according to the principles expressed in the Declaration of Helsinki.

\section{Conflict of interest}

No potential conflict of interest relevant to this article was reported.

\section{ORCID}

Bhavika Patel https://orcid.org/0000-0002-6799-3604

\section{REFERENCES}

1. British Orthopaedic Association. The care of patients with fragility fracture. 2007 [cited by 2019 Dec 4]. Available from: https://www.bgs.org.uk/sites/default/files/content/ attachment/2018-05-02/Blue\%20Book\%20on\%20fragility\%20fracture\%20care.pdf

2. van Staa TP, Dennison EM, Leufkens HG, et al. Epidemiology of fractures in England and Wales. Bone 2001;29:51722. http://dx.doi.org/10.1016/s8756-3282(01)00614-7.

3. National Institute for Health and Care Excellence. Osteoporosis: assessing the risk of fragility fracture. 2012 [cited by 2009 Dec 4]. Available from: https://www.nice.org.uk/ guidance/cg146

4. National Institute for Health and Care Excellence. Osteoporosis - prevention of fragility fractures. 2016 [cited by 2009 Dec 4]. Available from: https://cks.nice.org.uk/osteoporosis-prevention-of-fragility-fractures\#!scenario

5. National Institute for Clinical Excellence. Osteoporosis: assessment of fracture risk and the prevention of osteoporotic fractures in individuals at high risk. 2002 [cited by 2019 Dec 4]. Available from: https://www.nice.org.uk/ guidance/cg146/documents/osteoporosis-scope2

6. van Staa TP, Leufkens HG, Cooper C. Does a fracture at one site predict later fractures at other sites? A British cohort study. Osteoporos Int 2002;13:624-9. http://dx.doi.org/ 10.1007/s001980200084.

7. Burge RT, Worley $D$, Johansen A, et al. The cost of osteoporotic fractures in the UK: projections for 2000-2020. J Med Econ 2001;4:51-62. http://dx.doi.org/10.3111/200104051062. 8. National Osteoporosis Society. The Osteoporosis agenda England. 2015 [cited by 2019 Dec 4]. Available from: https: //theros.org.uk/media/1959/agenda-for-osteoporosisengland-final.pdf

9. Gausden EB, Nwachukwu BU, Schreiber JJ, et al. Opportunistic use of $\mathrm{CT}$ imaging for osteoporosis screening and bone density assessment: A qualitative systematic review. J Bone Joint Surg Am 2017;99:1580-90. http://dx.doi. org/10.2106/jbjs.16.00749.

10. Chou SH, LeBoff MS. Vertebral imaging in the diagnosis of osteoporosis: A clinician's perspective. Curr Osteoporos Rep 2017;15:509-20. http://dx.doi.org/10.1007/s11914017-0404-x.

11. Schreiber JJ, Kamal RN, Yao J. Simple assessment of global bone density and osteoporosis screening using standard radiographs of the hand. J Hand Surg Am 2017;42:244-9. http://dx.doi.org/10.1016/j.jhsa.2017.01.012.

12. Singh SV, Aggarwal H, Gupta V, et al. Measurements in mandibular pantomographic $\mathrm{X}$-rays and relation to skeletal mineral densitometric values. J Clin Densitom 2016;19: 255-61. http://dx.doi.org/10.1016/j.jocd.2015.03.004.

13. Barnett $E$, Nordin $B E$. The radiological diagnosis of osteoporosis: a new approach. Clin Radiol 1960;11:166-74. http: //dx.doi.org/10.1016/s0009-9260(60)80012-8.

14. Cootes TF, Hill A, Taylor CJ, et al. Use of active shape models for locating structure in medical images. Image Vis Comput 1994;12:355-65.

15. Navabi N, Motaghi R, Rezazadeh M, et al. Relationship between two panoramic radiography indices and bone mineral density of postmenopausal women with osteopenia and osteoporosis. J Dent (Shiraz) 2018;19:181-8. http:// dx.doi.org/10.30476/dentjods.2018.41810.

16. Aggarwal A, Panat SR. Identification of postmenopausal women at risk of osteoporosis using panoramic and intraoral radiographs- a review. Minerva Stomatol 2012;61: 323-8.

17. Alapati S, Reddy RS, Tatapudi R, et al. Identifying risk groups for osteoporosis by digital panoramic radiography. Contemp Clin Dent 2015;6:S253-7. http://dx.doi.org/10.4103/ 30976-237x.166833.

18. Mather J, MacDermid JC, Faber KJ, et al. Proximal humerus cortical bone thickness correlates with bone mineral density and can clinically rule out osteoporosis. J Shoulder Elbow Surg 2013;22:732-8. http://dx.doi.org/10.1016/j.jse. 2012.08.018.

19. Tingart MJ, Apreleva $M$, von Stechow $D$, et al. The cortical thickness of the proximal humeral diaphysis predicts bone 
mineral density of the proximal humerus. J Bone Joint Surg Br 2003;85:611-7. http://dx.doi.org/10.1302/0301620x.85b4.12843.

20. Damilakis J, Maris TG, Karantanas AH. An update on the assessment of osteoporosis using radiologic techniques. Eur Radiol 2007;17:1591-602. http://dx.doi.org/10.1007/ s00330-006-0511-z.

21. Kälvesten J, Lui LY, Brismar T, et al. Digital X-ray radiogram- metry in the study of osteoporotic fractures: Comparison to dual energy X-ray absorptiometry and FRAX. Bone 2016; 86:30-5. http://dx.doi.org/10.1016/j.bone.2016.02.011.

22. World Health Organization. WHO scientific group on the assessment of osteoporosis at primary health care level: Summary meeting report. 2007 [cited by 2020 Jul 22]. Available from: https://www.who.int/chp/topics/Osteoporosis.pdf 